\title{
Systemic Sarcoidosis Revealed by a Nasal Lesion: A Case Report
}

\author{
Madiha Mahfoudhi ${ }^{*}$, Khaled Khammassi2 ${ }^{2}$ Sami Turki1, Mamia Ben Salah² \\ ${ }^{1}$ Department of Internal Medicine A, Charles Nicolle Hospital, Tunis, Tunisia \\ ${ }^{2}$ Department of Oto-Rhino-Laryngology, Charles Nicolle Hospital, Tunis, Tunisia \\ Email: ${ }^{*}$ madiha mahfoudhi@yahoo.fr
}

Received 25 January 2015; accepted 12 April 2015; published 16 April 2015

Copyright (C) 2015 by authors and Scientific Research Publishing Inc.

This work is licensed under the Creative Commons Attribution International License (CC BY). http://creativecommons.org/licenses/by/4.0/

(c) (7) Open Access

\section{Abstract}

A 62-year-old man presented with bilateral nasal obstruction and recurrent epistaxis episodes. Physical examination revealed normal body temperature and free cervical lymph nodes areas. Nasal endoscopy found a congestive nasal mucosa associated to bilateral inferior turbinate hypertrophy with a granular aspect of the right inferior turbinate. The biological examination showed lymphopenia, inflammatory syndrome, high serum level of angiotensin-converting enzyme and $\beta 2$ microglobulin. Tuberculin skin test and Quantiferon gold were negative. The nasal and accessory salivary gland biopsies revealed granulomatous giant cell lesions non-caseating evoking systemic sarcoidosis. Ziehl-Neelsen staining and Lowenstein culture were negative. The diagnosis was systemic sarcoidosis revealed by nasal localization. The systemic extension research was negative. The treatment was based on corticosteroids $(1 \mathrm{mg} / \mathrm{kg} / \mathrm{day})$ and gradual degression doses associated to a local nasal corticosteroid. The outcome was favorable with improvement of nasal obstruction and disappearance of initial lesions. The follow-up was 2 years.

\section{Keywords}

\section{Sarcoidosis, Nose, Granuloma, Corticosteroids}

\section{Introduction}

Sarcoidosis is a systemic non-caseous granulomatous disease of non elucidated etiology [1]-[3]. It involves more frequently the mediastinum, lungs, skin and lymph nodes [1]. Typical lesions confirmed by histological examination consist of epitheloid and gigantocellular granulomas without caseous necrosis.

"Corresponding author.

How to cite this paper: Mahfoudhi, M., Khammassi, K., Turki, S. and Salah, M.B. (2015) Systemic Sarcoidosis Revealed by a Nasal Lesion: A Case Report. International Journal of Clinical Medicine, 6, 257-261. 
Uveitis, neurological feature, interstitial pneumonitis, pulmonary fibrosis, cardiomyopathy, hypercalcemia, endocrine manifestations, sicca syndrome and renal disturbance may be revelatory features of the systemic sarcoidosis [1] [2].

Elevated serum levels of angiotensin-converting enzyme and serum $\beta 2$ microglobulin aren't pathognomonic of sarcoidosis diagnostic since they can be encountered in many other granulomatous pathologies. It's an exclusion diagnosis [1]-[3].

The diagnosis depends on the association of clinical, biological, radiological and histological elements. The elimination of infectious causes such as tuberculosis, autoimmune and tumoral diseases is an indispensable step.

Thoracic radiologic anomalies such as bilateral mediastinal lymphadenopathy or diffuse micronodular pulmonary infiltration occur frequently in sarcoidosis [1]-[4].

A diagnosis difficulty is encountered in case of extrathoracic revelatory disturbance. Nasal localization is rare and represents less than $2 \%$ of cases. The clinical symptoms are not specific of sarcoidosis inducing a diagnostic lateness [5].

In half of cases, sarcoidosis resolves spontaneously within 2 years. Whereas refractory chronic forms of sarcoidosis were described leading to pulmonary and extrapulmonary fibrosis and organs impairment [1]-[4]. Multiple indicators of prognosis have been proposed [4].

The aim of this study is to demonstrate through this rare case and the literature the diagnosis difficulty in case of isolate nasal involvement, to determine therapeutic modalities and prognosis profile.

\section{Case Report}

A 62-year-old man, with no particular history, presented bilateral nasal obstruction and recurrent epistaxis episodes lasting for one year. He did not have a tuberculosis contagious context. Anamnesis found the notion of xerostomia which was overlooked by the patient.

Physical examination revealed normal body temperature and pulse rate and a blood pressure of 130/80 mmHg. The pulmonary, cardiac, abdominal, neurological, and ophtalmological examinations were without abnormalities. The electrocardiogram was without anomaly. Urine dipstick was normal. Cervical lymph nodes areas were free.

Nasal endoscopy found a congestive nasal mucosa with crusts associated to bilateral inferior turbinate hypertrophy with a granular aspect of the right inferior turbinate (Figure 1).

The biological examination showed lymphopenia, inflammatory syndrome with erythrocyte sedimentation rate of $44 \mathrm{~mm} / \mathrm{h}$. Blood cell counts, hemoglobin level, liver and renal function tests, serum and urine calcium levels, serum electrolytes levels were within the normal range.

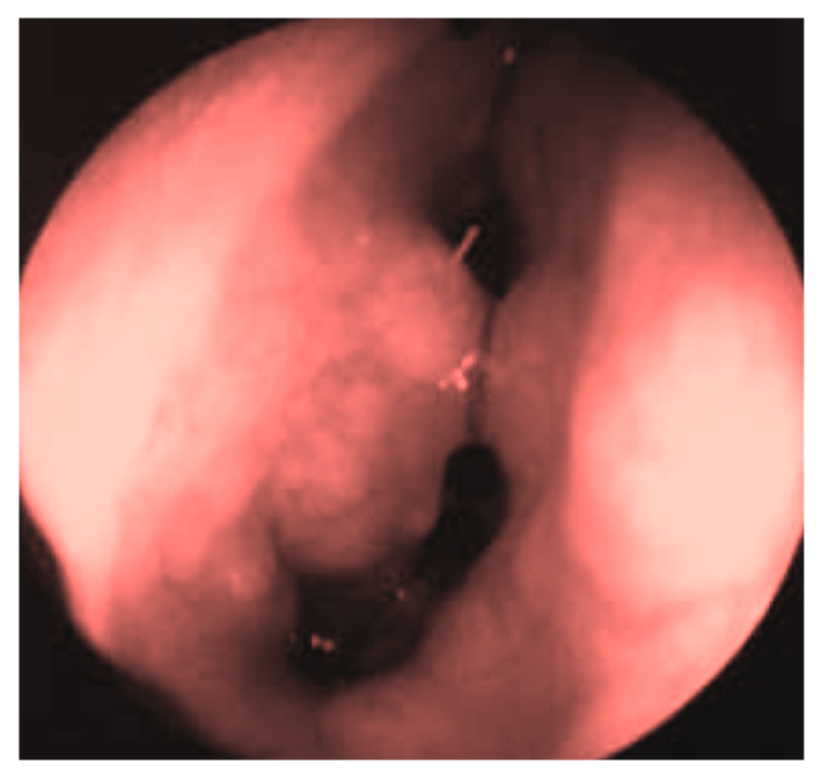

Figure 1. Bilateral inferior turbinate's hypertrophy with granular aspect of the right inferior turbinate. 
The serum angiotensin-converting enzyme level was $224 \mathrm{U} / \mathrm{L}$ (normal values: $<60 \mathrm{U} / \mathrm{L}$ ). Serum $\beta 2$ microglobulin corresponding to $3.8 \mathrm{mg} / \mathrm{L}$ was also elevated (normal values: 0.9 to $2.5 \mathrm{mg} / \mathrm{L}$ ).

Sinus CT scan was normal.

Chest X-ray and CT scan was without anomalies. Full lung function tests, monooxide gas-transfer factor, bronchoscopy and bronchoalveolar lavage were normal. The kidney echography was normal.

Otherwise, tuberculin skin test and Quantiferon gold were negative.

The histological examination of nasal and accessory salivary gland biopsies revealed granulomatous giant cell lesions non-caseating evoking systemic sarcoidosis. Ziehl-Neelsen staining and Lowenstein culture were negative eliminating tuberculosis.

The diagnosis was systemic sarcoidosis revealed by nasal localization.

The systemic extension research, involving particularly lungs, mediastinum, skin, nodes, bones, kidneys and heart, was negative.

The treatment was based on corticosteroids ( $1 \mathrm{mg} / \mathrm{kg} / \mathrm{day})$ and gradual degression doses associated to a local nasal corticosteroid.

The outcome was favorable with improvement of nasal obstruction and disappearance of initial lesions. The follow-up was 2 years.

\section{Discussion}

Neck mass, parotid swelling and facial nerve palsy are the most frequent complaints in cases of head and neck sarcoidosis, isolated nasosinusal involvement of the disease is rare (1\% of cases) inducing a lateness of diagnosis delay [6].

Nasosinusal sarcoidosis may be revelatory, isolated or associated with multisystemic sarcoidosis.

Functional signs are nonspecific represented mainly by nasal obstruction, purulent discharge, rarely epistaxis.

Physical examination may objectify an aspect rhinitis or rhinosinusitis sometimes crusty chronicle of turbinoseptal synechiae and especially nodular lesions in the turbinates. Mucosal lesions can be associated to macular, popular or nodular skin lesions.

Mallis et al. reported a case of a 46-year-old woman presenting a nasal congestion over a six month period with occasional purulent discharge occurring in the last month [6].

Dessouky presented a 20-year-old female who complained of nasal obstruction [7].

Two cases of nasosinusal sarcoidosis associated to multisystemic sarcoidosis were reported by Kort. Both patients were females aged over 43 years. The primary symptom was also a nasal obstruction [8].

Crusting and nasal obstruction may be revelatory of the sarcoidosis inducing a lateness of the diagnosis delay [9]. The low incidence of this disease may be due to unawareness of this pathology especially in case of isolated non habitual involvement as nasal localization [9].

Lupus pernio is a typical cutaneous lesion of the sarcoidosis located in the face and the nose. Our patient hadn't this lesion which is highly suggestive of the positive diagnosis and accessible to biopsy [10].

Anterior rhinoscopy objectified hypertrophy of nasal mucosa, purplish discoloration and granulomatous aspect of the inferior turbinates in a case of nasal sarcoidosis [6].

The nasal endoscope allows an early diagnosis in case of nasal lesion due to sarcoidosis [9].

Meybeck et al. underwent a prospective study which had compiled 62 patients affected with sarcoidosis histologically confirmed. Among them, 38 patients reported at least one nasal symptom. A non specific abnormality of the CT scan was reported in 70\% of cases. Rhinoscopy showed a mucosal lesion in 11 patients. The presence of mucosal nodules on the turbinates was shown in 4 cases with histological sarcoidosis confirmation (6.5\%) [11]. Authors concluded that involvement of the nasal sinuses is rare; anterior rhinoscopy permits diagnosis before the appearance of typical lesions and guides biopsies [11].

The biological examination reveals a high serum level of angiotensin-converting enzyme and $\beta 2$ microglobulin which are not pathognomonic of sarcoidosis.

CT imaging has a particular interest in the positive and differential diagnosis.

A nasal mass involving the ethmoid sinuses with destruction of the cribriform plate and intracranial extension was reported in a case of sarcoidosis [7].

In our case, there was no extension to cranial or cerebral structures.

Some diagnoses should be mentioned, such as tuberculosis, Wegener's disease, actinomycosis, Crohn's dis- 
ease; since nasal sarcoidosis can mimic feature of various pathologies. Tuberculosis should be eliminated in endemic countries because of its high prevalence and the potential aggravation under a wrong corticosteroids therapy indicated for a false diagnosis of sarcoidosis. Research of Mycobacterium tuberculosis, tuberculin skin test, Quantiferon gold, Ziehl-Neelsen staining and Lowenstein culture must be negative.

Tomodensitometric and guided biopsy results are the key diagnosis.

Histological examination of biopsies from the nasal lesion led to a diagnosis of non-caseating granulomas in several reported cases of nasal sarcoidosis [5]-[9]. Nasal biopsy is a key for the positive diagnosis of sarcoidosis showing suggestive lesions with absence of caseous necrosis.

Braun et al. had reported 13 cases of nasosinusal sarcoidosis. One case had isolated nasosinusal lesions and 12 cases were associated with other lesions of the chest, skin, liver, spleen, bone, eyes, salivary glands, peripheral lymph nodes or with neurosarcoidosis. The clinical and CT presentations were various and non specific. Nasal biopsy guided by physical examination was easy and an essential element for the positive diagnosis [12].

Where mucosal changes are present within the nose, biopsy gives a high diagnostic yield [13].

The special feature of our case is to have a symptomatic and revealing nasal lesions associated to overlooking xerostomia without sinusal or skinny involvement.

This observation showed the diagnosis difficulty in case of isolated nasal disturbances. Sarcoidosis should be considered after eliminating tuberculosis if the patient lives in endemic country.

Treatment of nasosinusal sarcoidosis is essentially medical. There is no standard treatment or clear consensus to follow.

It is classically based on local and systemic corticosteroids therapy with gradually reduced doses, while hydroxychloroquine or methotrexate is given in the cortico-resistant forms [5]-[11]. The aim of treatment is to have clinical control with the lowest dose of steroids in order to avoid adverse effects.

Our patient had not presented recurrence or drug's side effects.

Hermet et al. had demonstrated infliximab efficiency as treatment of refractory nasosinusal sarcoidosis to corticosteroids and methotrexate in a series of three cases [14].

Surgical treatment is indicated in the presence of sequelae or disfigurement.

The recurrence is frequent after tapering off or interrupting corticosteroids with several side effects.

Despite of a well conducted treatment, the evolution can be marked by recurrence and complications such as fibrosis and adhesions.

\section{Conclusion}

The medical treatment of nasal sarcoidosis should be administered early to prevent progression to fibrosis and sequelae. This requires considering this diagnosis in case of any unusual and unexplained chronic rhinologic symptomatology.

\section{Disclosure Statement}

The authors have nothing to disclose. Informed consent was obtained from the patient to report this case.

\section{References}

[1] Baughman, R.P., Lower, E.E. and du Bois, R.M. (2003) Sarcoidosis. Lancet, 361, 1111-1118. http://dx.doi.org/10.1016/S0140-6736(03)12888-7

[2] Criado, E., Sanchez, M., Ramirez, J., Arguis, P., de Caralt, T.M., Perea, R.J., et al. (2010) Pulmonary Sarcoidosis: Typical and Atypical Manifestations at High-Resolution CT with Pathologic Correlation. Radiographics, 30, 15671586. http://dx.doi.org/10.1148/rg.306105512

[3] Iannuzzi, M.C. and Fontana, J.R. (2011) Sarcoidosis: Clinical Presentation, Immunopathogenesis, and Therapeutics. JAMA, 305, 391-399. http://dx.doi.org/10.1001/jama.2011.10

[4] Neville, E., Walker, A.N. and James, D.G. (1983) Prognostic Factors Predicting the Outcome of Sarcoidosis: An Analysis of 818 Patients. QJM, 52, 525-533.

[5] Khammassi, N., Kwas, H., Bayoudh, A., Abdelhedi, H., Zendah, I., Cherif, I., et al. (2014) Systemic Sarcoidosis Revealed by an Involvement of the Nasal Sinuses. La Tunisie Médicale, 92, 181-183.

[6] Mallis, A., Mastronikolis, N.S., Koumoundourou, D., Stathas, T. and Papadas, T.A. (2010) Sinonasal Sarcoidosis. A Case Report. European Review for Medical and Pharmacological Sciences, 14, 1097-1099. 
[7] Dessouky, O.Y. (2008) Isolated Sinonasal Sarcoidosis with Intracranial Extension: Case Report. Acta Otorhinolaryngologica Italica, 28, 306-308.

[8] Kort, K.R., Cherif, F., Mokni, M., Bouraoui, S., Mnif, E., Sahtout, S., et al. (2006) Nasosinusal Sarcoidosis: 2 Cases. A Report of 2 Cases. La Tunisie Médicale, 84, 48-51.

[9] Morre, T.D., Hamels, K., Clement, P.A. and Bourgain, C. (1998) Nasal Mucosal Sarcoidosis: A Case Report. Acta Oto-Rhino-Laryngologica Belgica, 52, 75-77.

[10] Khachemoune, A. (2006) Papules and Plaques on the Nose. Lupus Pernio. American Family Physician, 73, $1431-1432$.

[11] Meybeck, A., Just, N., Heurtebise, F., Chanez, P., Crampette, L., Darras, J., et al. (2004) Involvement of the Nasal Sinuses in Sarcoidosis. A Prospective Study of 63 Patients. Revue des Maladies Respiratoires, 21, 279-286. http://dx.doi.org/10.1016/S0761-8425(04)71286-3

[12] Braun, J.J., Bourjat, P., Oster, J.P., Pauli, G., Quoix, E. and Gentine, A. (2001) Nasosinusal Sarcoidosis: 13 Cases. Annales d'Otolaryngologie et de Chirurgie Cervico-Faciale, 118, 238-244.

[13] Fergie, N., Jones, N.S. and Havlat, M.F. (1999) The Nasal Manifestations of Sarcoidosis: A Review and Report of Eight Cases. Journal of Laryngology Otology, 113, 893-898. http://dx.doi.org/10.1017/S0022215100145529

[14] Hermet, M., le Guenno, G., Rieu, V., Philippe, P. and Ruivard, M. (2012) Nasosinusal and Cervical Sarcoidosis: A Case Series of Three Patients and Literature Review. La Revue de Médecine Interne, 33, 46-49. http://dx.doi.org/10.1016/j.revmed.2011.04.002 